\title{
Analysis of Influencing Factors of Heat Load of Heating Power Station
}

\author{
$\mathrm{Yu} \mathrm{Lu}{ }^{1}, \mathrm{Rui}_{\mathrm{Li}^{1 *}}$ \\ ${ }^{1}$ School of Environment and Energy Engineering, Beijing University of Civil Engineering and Architecture, Beijing 100044, China
}

\begin{abstract}
Heating system load forecasting is very important in heating system planning and formulating heating plans. More accurate load forecasting can obtain greater economic and social benefits for the heating power station. The selection of influencing factors affecting heating load forecasting has a decisive effect on the accuracy of load forecasting. Based on the analysis of heat load of typical heating power station, this paper compares and analyses the influence of outdoor temperature, wind speed and load of previous days, etc. The main factors that should be considered in heating load forecasting are obtained.
\end{abstract}

\section{Introduction}

At present, buildings have become the largest energy consumption body, accounting for more than one-third of global energy consumption, and the heating energy consumption of buildings accounts for $25 \%$ of its proportion, which undoubtedly increases the burden of urban thermal power stations. Therefore, the on-demand supply of heating load has practical significance for reducing the energy consumption of thermal power stations [1]. With the popularization of building automation, a large amount of data has also begun to be stored. These data can reflect the actual operating characteristics of the building. Through the application and analysis of the data, it can optimize the control of building energy management [2]. Especially for residential quarters, the application analysis of historical data has guiding value for energy-saving heating, and at the same time has practical significance for improving residents' satisfaction with heating. In recent years, researchers have used data analysis to carry out load forecasting in various fields such as heating systems and power systems to achieve energy conservation and emission reduction research. Li Rui et al. [3] used BP neural network algorithm to analyse the influencing factors of residential district heating compliance and improve the prediction accuracy in the residential district heating load research, also analyses prediction methods of the thermal load of urban thermal power stations and proposed a prediction method. Li Siqi et al. [4] focused on analysing the influencing factors of various outdoor conditions, combined with neural network and genetic algorithm to optimize the heating system, and basically realized the possibility of heating on demand. This paper focused on the analysis and comparison of the influencing factors affecting the heating load. The outdoor meteorological conditions and heating conditions of the heating station are mainly investigated and compared, and the factors affecting the load of the heating station are analysed in detail, so as to obtain the main factors that should be considered in the heating load forecasting.

\section{Analysis of Factors Affecting Heating Load of Heating Power Station}

\subsection{Meteorological factors and analysis}

\subsubsection{Outdoor temperature}

The outdoor temperature has a periodic change between day and night and the temperature difference is large, with the maximum temperature difference reaching $15.6^{\circ} \mathrm{C}$. As shown in Figure 1, the maximum and minimum temperature change curves for 10 consecutive days in February 2020 . The heating load is greatly affected by the outdoor temperature in the research on the heating load prediction of the residential area, which is a necessary input parameter for the heating load prediction. Figure 2 is a graph based on the outdoor temperature and heating load of a heating station for 10 consecutive days in winter. It can clearly see the change trend of the heating load with the outdoor temperature, namely: When the outdoor environment temperature is low, the heating load is larger; when the outdoor environment temperature is high, the heating load is smaller, showing a negative correlation in general. 


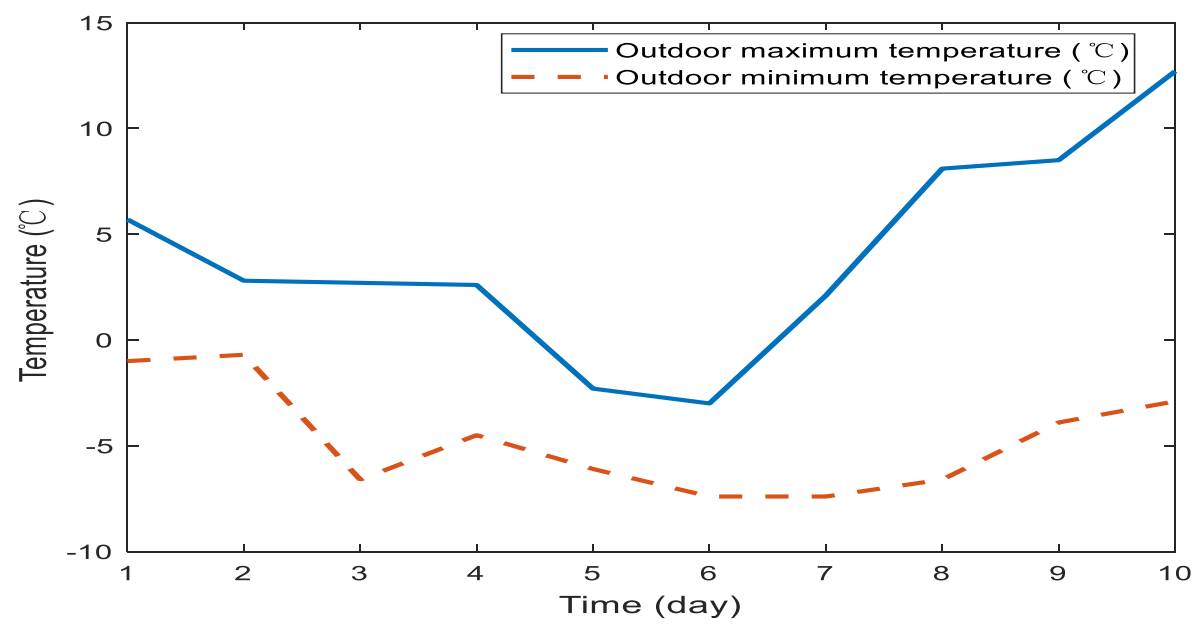

Figure 1. Relationship between outdoor maximum temperature and outdoor minimum temperature

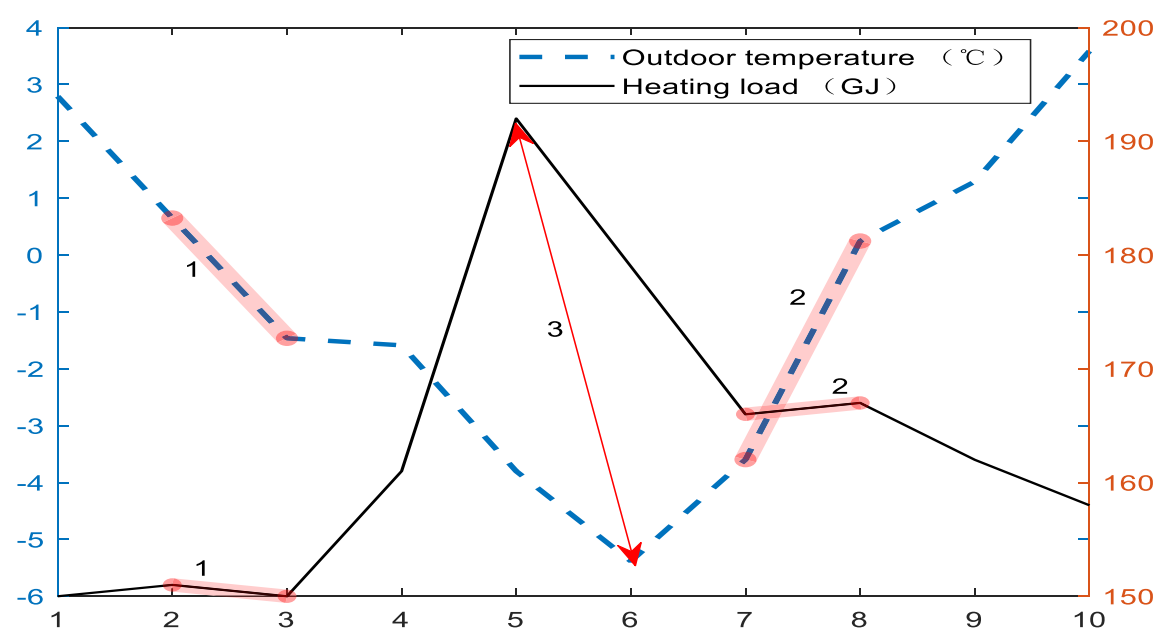

Figure 2. Relationship between heating load and outdoor temperature.

In the same way, the heating load is also roughly negatively correlated with the highest outdoor temperature and the lowest outdoor temperature. Therefore, the outdoor temperature sequence is used as a reference factor.

\subsubsection{Wind speed}

The wind has the ability to carry heat, and the change of outside wind speed will affect the real outdoor temperature. When the wind speed increases, the real outdoor temperature value will be lower than the measured outdoor temperature value. In studying the application analysis of load forecasting control methods for room temperature and climate compensation, Feng Guoyan et al. [5] presented the correction amount of wind speed to temperature in the form of a formula. Therefore, when studying the relationship between outdoor temperature and the heat load of the central heating system, it is necessary to consider the influence of wind speed on outdoor temperature, and it can be seen from Figure 3 that there is a certain correlation between wind speed and heating load. Therefore, outdoor wind speed is used as an influencing factor for load forecasting. 


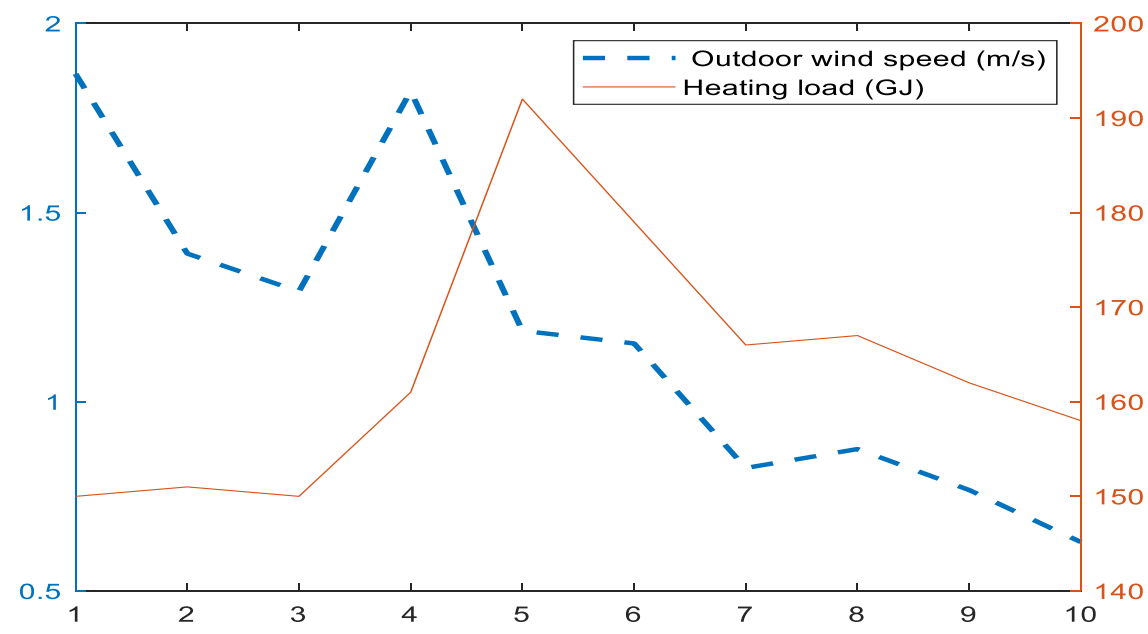

Figure 3. Relationship between heating load and outdoor wind speed

\subsection{System factors and analysis}

Through the outdoor temperature and heating load trend line in Figure 2, it is not difficult to see that the temperature drops, but the heat supply is almost constant or even drops on some days, as shown by the marking point 1; Although the temperature rises, the heat supply is almost constant or even rises on some days, as indicated by mark 2; It can be seen from marking point 3 that when the outdoor temperature is at the lowest point, the heating load is not at the highest point. Therefore, the factors that affect the heating load of a residential area are not only affected by the outdoor temperature, but also by other factors, such as the heat storage capacity of the envelop configuration of buildings and indoor furniture facilities and the delay and attenuation characteristics of heating load, etc. Partial data of outdoor temperature and heating load are shown in Table 1. Li Rui et al. [1] proved in the research on heating load forecast of residential area that the factors that affect the heating load of residential area are not only the outdoor temperature, but also the influence of system factors such as heating load of the previous few days, heat consumption and building characteristics, etc. Therefore, the heat load value of the first three days is also listed as an influencing factor.

Table 1. Partial data of outdoor temperature and heating load

\begin{tabular}{ccc}
\hline Date & $\begin{array}{c}\text { February } 2 \text { to } \\
\text { February } 3\end{array}$ & $\begin{array}{c}\text { February } 7 \text { to } \\
\text { February } 8\end{array}$ \\
\hline Outdoor & 0.65 decrease to - & -3.60 rise to 0.25 \\
temperature $\left({ }^{\circ} \mathrm{C}\right)$ & 1.46 & 166 rise to 167 \\
Heating load $(\mathrm{GJ})$ & 151 decrease to \\
& 150 & \\
\hline
\end{tabular}

\section{Verification of influencing factors}

Due to the linear and non-linear relationship between influencing factors and heating load, this paper only uses SCE-UA algorithm to verify the linear relationship between influencing factors and heating load. Figure 4 shows that the scattered points are basically distributed around the 1:1 line with a higher accuracy: $\mathrm{R}^{2}=0.82$. This shows that the above influencing factors can be used as load forecasting factors.

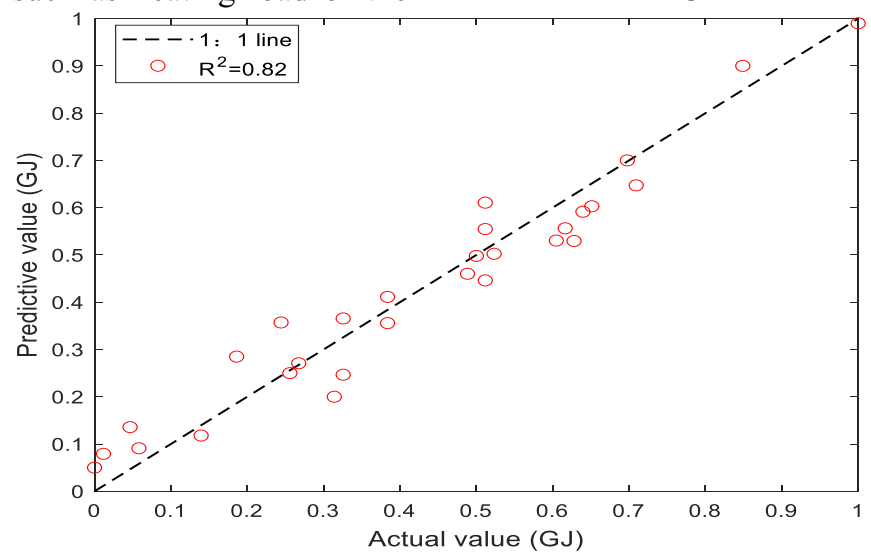

Figure 4. Scatter plot of predicted and actual values 


\section{Conclusion}

According to the research and analysis in this paper, the conclusions are obtained as follows:

- The heating load is roughly negatively correlated with the highest outdoor temperature and the lowest outdoor temperature. Outdoor temperature sequence is used as a reference factor of heating load.

- $\quad$ There is a certain correlation between wind speed and heating load. Outdoor wind speed is used as an influencing factor for load forecasting. When researching the relationship between outdoor temperature and the heat load of the central heating system, it is necessary to consider the influence of wind speed on apparent temperature and appropriate correction of outdoor calculated temperature.

- The factors that affect the heating load of a residential area are not only affected by the outdoor temperature, but also by other factors, such as the heat storage capacity of the envelop configuration of buildings and indoor furniture facilities and the delay and attenuation characteristics of heating load.

- $\quad$ Outdoor temperature series, outdoor wind speed and heating load in the previous three days are used as the influencing factors, the result of SCEUA algorithm shows that there is a significant correlation between the influencing factors and heating load.

\section{Acknowledgments}

This paper was supported by 'Beijing Advanced Innovation Center For Future Urban Design, Beijing University Of Civil Engineering And Architecture' (UDC2019011121).

\section{References}

1. Li R, Dong Y, Xue W, Fu B. (2019) Research on Heating Load Forecast in Residential Area. J. District Heating, 28: 31+60.

2. Shan X W. (2020) Principle analysis of energysaving control technology used in intelligent buildings $[\mathrm{J}]$. Technology Wind, 9.

3. Li R, Hao X J, Fu B, Wang L X, Sun Z Y, Dong Y. (2018) Analysis and prediction of factors affecting Heating Load of Urban Thermal Station. J. District Heating, 25-29.

4. Li S Q, Jiang Z J. (2019) Research on energy saving control system of heating station. J. Modern Electronics Technique, 42:126-130.

5. Feng G Y, Zou R Y, Wang C Y, Jiang X, Zheng J L, Bao X B. (2019) Application Analysis of Load Predictive Control Method Based on Room Temperature and Climate Compensation. J. District Heating, 12-20. 